TRANSACTIONS OF THE

AMERICAN MATHEMATICAL SOCIETY

Volume 364, Number 4, April 2012, Pages 2227-2240

S 0002-9947(2011)05571-6

Article electronically published on December 1, 2011

\title{
THE COMPLEX CROWN FOR HOMOGENEOUS HARMONIC SPACES
}

\author{
ROBERTO CAMPORESI AND BERNHARD KRÖTZ
}

\begin{abstract}
The complex crown of a noncompact Riemannian symmetric space $X=G / K$ is generalized to the case of homogeneous harmonic spaces $S=$ $N A$. We prove that every eigenfunction of the Laplace-Beltrami operator on $S$ extends holomorphically to the crown, and that the crown is the maximal

$S$-invariant domain in $S_{\mathbb{C}}$ with this property.
\end{abstract}

\section{INTRODUCTION}

Let $X$ be a simply connected homogeneous harmonic Riemannian space. Then according to [7], Corollary 1.2, $\mathrm{X}$ is isometric (up to scaling of the metric) to one of the following spaces:

(i) $\mathbb{R}^{n}$.

(ii) $S^{n}, P^{k}(\mathbb{C}), P^{l}(\mathbb{H})$, or $P^{2}(\mathbb{O})$, i.e., a compact rank one symmetric space.

(iii) $H^{n}(\mathbb{R}), H^{k}(\mathbb{C}), H^{l}(\mathbb{H})$, or $H^{2}(\mathbb{O})$, i.e., a noncompact rank one symmetric space.

(iv) A solvable Lie group $S=A \ltimes N$, where $N$ is of Heisenberg-type and $A \simeq \mathbb{R}^{+}$acts on $N$ by anisotropic dilations preserving the grading.

We denote by $\mathcal{L}$ the Laplace-Beltrami operator on $X$. In case (i), $\mathcal{L}$-eigenfunctions on $\mathbb{R}^{n}$ extend to holomorphic functions on $\mathbb{C}^{n}$. Likewise, in case (ii), $\mathcal{L}$ eigenfunctions on $X=U / K$ admit a holomorphic continuation to the whole affine complexification $X_{\mathbb{C}}=U_{\mathbb{C}} / K_{\mathbb{C}}$ of $X$. This is no longer true in case (iii). However, in this case, and more generally for a noncompact Riemannian symmetric space of any rank $X=G / K$, there exists a unique $G$-invariant domain $\operatorname{Cr}(X)$ of $X_{\mathbb{C}}=G_{\mathbb{C}} / K_{\mathbb{C}}$ containing $X$, the complex crown, with the following property ([13, 11]):

Every $\mathcal{L}$-eigenfunction on $X$ admits a holomorphic extension to $\operatorname{Cr}(X)$, and this domain is maximal for this property.

The objective of this paper is to obtain an analogous theory for the spaces in (iv) above.

We note that all spaces in (iii), except for $H^{n}(\mathbb{R})$, fall into class (iv) by identifying the symmetric space $X=G / K$ with the $N A$-part in the Iwasawa decomposition $G=N A K$ of a noncompact simple Lie group $G$ of real rank one, and by suitably scaling the metric.

Our investigations start with a new model of the crown domain for the rank one symmetric spaces $X$. We describe $\operatorname{Cr}(X)$ in terms of the Iwasawa coordinates $A$ and $N$ only. Henceforth we refer to this new model as the mixed model of the

Received by the editors April 22, 2010 and, in revised form, August 17, 2010, December 14, 2010, February 1, 2011 and February 15, 2011.

2010 Mathematics Subject Classification. Primary 22E25, 22E46, 43 A85.

Key words and phrases. Homogeneous harmonic spaces, complex crown.

(C)2011 American Mathematical Society 
crown. In section 2 we provide the mixed model for the two basic cases, i.e., the symmetric spaces associated with the groups $G=\operatorname{Sl}(2, \mathbb{R})$ and $G=\mathrm{SU}(2,1)$.

Starting from the two basic cases, reduction of symmetry allows us to obtain a mixed model for all rank one symmetric spaces and motivates a definition of $\operatorname{Cr}(S)$ for the remaining spaces in (iv). This is worked out in section 3 .

Finally, in section 4, we use recent results from 11 to prove the holomorphic extension of $\mathcal{L}$-eigenfunctions on $S$ to the crown domain $\operatorname{Cr}(S)$ and establish maximality of $\operatorname{Cr}(S)$ with respect to this property.

\section{MiXed MODEL FOR THE CROWN DOMAin}

The crown domain can be realized inside the complexification of an Iwasawa $A N$-group. The goal of this section is to make this explicit for the rank one groups $\mathrm{Sl}(2, \mathbb{R})$ and $\mathrm{SU}(2,1)$.

2.1. Notation for rank one spaces. Let $G$ be a connected semi-simple Lie group of real rank one. We assume that $G \subset G_{\mathbb{C}}$, where $G_{\mathbb{C}}$ is the universal complexification of $G$, and that $G_{\mathbb{C}}$ is simply connected. We fix an Iwasawa decomposition $G=N A K$ and form the Riemannian symmetric space

$$
X=G / K \text {. }
$$

The Cartan involution $\theta$ on $G$ associated to $K$ extends holomorphically to $G_{\mathbb{C}}$, say $\theta_{\mathbb{C}}$, and the $\theta_{\mathbb{C}}$-fixed points will be denoted by $K_{\mathbb{C}}$. We arrive at a totally real embedding

$$
X \hookrightarrow X_{\mathbb{C}}:=G_{\mathbb{C}} / K_{\mathbb{C}}, \quad g K \mapsto g K_{\mathbb{C}} .
$$

Let $x_{0}=K \in X$ be the base-point.

Let $\mathfrak{g}, \mathfrak{k}, \mathfrak{a}$ and $\mathfrak{n}$ be the Lie algebras of $G, K, A$ and $N$. Let $\Sigma^{+}=\Sigma(\mathfrak{a}, \mathfrak{n})$ be the set of positive roots and put

$$
\Omega:=\left\{Y \in \mathfrak{a}\left|\forall \alpha \in \Sigma^{+}\right| \alpha(Y) \mid<\pi / 2\right\} .
$$

Note that $\Omega$ is a symmetric interval in $\mathfrak{a} \simeq \mathbb{R}$. The crown domain of $X$ is defined as

$$
\operatorname{Cr}(X):=G \exp (i \Omega) \cdot x_{0} \subset X_{\mathbb{C}} .
$$

Let us point out that $\Omega$ is invariant under the Weyl group $\mathcal{W}=N_{K}(A) / Z_{K}(A) \simeq \mathbb{Z}_{2}$ and that $\exp (i \Omega)$ consists of elliptic elements in $G_{\mathbb{C}}$. We will refer to (2.1) as the elliptic model of $\operatorname{Cr}(X)$ (see [13] for the basic structure theory in these coordinates).

Let us define a domain $\Lambda$ in $\mathfrak{n}$ by

$$
\Lambda:=\left\{Y \in \mathfrak{n} \mid \exp (i Y) \cdot x_{0} \in \operatorname{Cr}(X)\right\}_{0},
$$

where $\{\cdot\}_{0}$ refers to the connected component of $\{\cdot\}$ which contains 0 .

The set $\Lambda$ is explicitly determined in [10, Th. 8.11. Further by [10, Th. 8.3, we have

$$
\operatorname{Cr}(X)=G \exp (i \Lambda) \cdot x_{0} .
$$

We refer to (2.2) as the unipotent model of $\operatorname{Cr}(X)$.

Finally let us mention the fact that $\operatorname{Cr}(X) \subset N_{\mathbb{C}} A_{\mathbb{C}} \cdot x_{0}$, which brings us to the question of whether $\operatorname{Cr}(X)$ can be expressed in terms of $A, N, \Omega$ and $\Lambda$. This is indeed the case and will be considered in the following two subsections for the groups $\mathrm{Sl}(2, \mathbb{R})$ and $\mathrm{SU}(2,1)$. 


\subsection{Mixed model for the upper half plane. Let}

$$
G=\operatorname{Sl}(2, \mathbb{R}) \quad \text { and } \quad G_{\mathbb{C}}=\operatorname{Sl}(2, \mathbb{C}) .
$$

Our choices of $A, N$ and $K$ are as follows:

$$
\begin{aligned}
A & =\left\{a_{t}=\left(\begin{array}{cc}
t & 0 \\
0 & 1 / t
\end{array}\right) \mid t>0\right\}, \\
A_{\mathbb{C}} & =\left\{a_{z}=\left(\begin{array}{cc}
z & 0 \\
0 & 1 / z
\end{array}\right) \mid z \in \mathbb{C}^{*}\right\}, \\
K & =\operatorname{SO}(2, \mathbb{R}), \quad K_{\mathbb{C}}=\operatorname{SO}(2, \mathbb{C}),
\end{aligned}
$$

and

$$
\begin{aligned}
N & =\left\{n_{x}=\left(\begin{array}{ll}
1 & x \\
0 & 1
\end{array}\right) \mid x \in \mathbb{R}\right\}, \\
N_{\mathbb{C}} & =\left\{n_{z}=\left(\begin{array}{ll}
1 & z \\
0 & 1
\end{array}\right) \mid z \in \mathbb{C}\right\} .
\end{aligned}
$$

We will identify $X=G / K$ with the upper half plane $\mathbf{H}=\{z \in \mathbb{C} \mid \operatorname{Im} z>0\}$ via the map

$$
X \rightarrow \mathbf{H}, \quad g K \mapsto \frac{a i+b}{c i+d} \quad\left(g=\left(\begin{array}{ll}
a & b \\
c & d
\end{array}\right)\right) .
$$

Note that $x_{0}=i$ within our identification.

We view $X=\mathbf{H}$ inside of the complex projective space $\mathbb{P}^{1}(\mathbb{C})=\mathbb{C} \cup\{\infty\}$ and note that $\mathbb{P}^{1}(\mathbb{C})$ is homogeneous for $G_{\mathbb{C}}$ with respect to the usual fractional linear action:

$$
g(z)=\frac{a z+b}{c z+d} \quad\left(z \in \mathbb{P}^{1}(\mathbb{C}), g=\left(\begin{array}{ll}
a & b \\
c & d
\end{array}\right) \in G_{\mathbb{C}}\right) .
$$

Let us remark that the map

$$
X_{\mathbb{C}} \rightarrow \mathbb{P}^{1}(\mathbb{C}) \times \mathbb{P}^{1}(\mathbb{C}) \backslash \text { diag, } \quad g K_{\mathbb{C}} \mapsto(g(i), g(-i))
$$

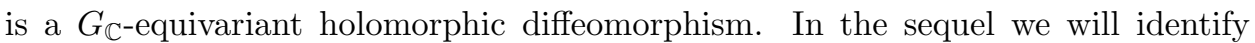
$X_{\mathbb{C}}$ with $\mathbb{P}^{1}(\mathbb{C}) \times \mathbb{P}^{1}(\mathbb{C}) \backslash$ diag, and emphasize that within this identification the embedding of (2.3) becomes

$$
X \hookrightarrow X_{\mathbb{C}}, \quad z \mapsto(z, \bar{z}) .
$$

We will denote by $\bar{X}$ the lower half plane, and note that the crown domain for $\mathrm{Sl}(2, \mathbb{R})$ is given by

$$
\operatorname{Cr}(X)=X \times \bar{X} \text {. }
$$

We note that

$$
\Omega=\left\{\left(\begin{array}{cc}
x & 0 \\
0 & -x
\end{array}\right) \mid x \in(-\pi / 4, \pi / 4)\right\}
$$

and

$$
\Lambda=\left\{\left(\begin{array}{ll}
0 & x \\
0 & 0
\end{array}\right) \mid x \in(-1,1)\right\} .
$$

With that we come to the mixed model for the crown which combines both parametrizations in an unexpected way.

We let $F:=\{ \pm \mathbf{1}\}$ be the center of $G$ and note that

$$
N_{\mathbb{C}} A_{\mathbb{C}} \cdot x_{0}=\mathbb{C} \times \mathbb{C} \backslash \text { diag, }
$$


and

$$
N_{\mathbb{C}} A_{\mathbb{C}} \cdot x_{0} \simeq N_{\mathbb{C}} A_{\mathbb{C}} / F .
$$

Proposition 2.1. Let $G=\mathrm{Sl}(2, \mathbb{R})$. Then the map

$$
N A \times \Omega \times \Lambda \rightarrow \operatorname{Cr}(X), \quad(n a, H, Y) \mapsto n a \exp (i H) \exp (i Y) \cdot x_{0}
$$

is an AN-equivariant diffeomorphism.

Proof. By the facts listed above we only have to show that the map is defined and onto. For that we first note that

$$
\exp (i \Lambda) \cdot x_{0}=\{((1+t) i,-(1-t) i) \mid t \in(-1,1)\},
$$

and thus

$$
A \exp (i \Lambda)=i \mathbb{R}^{+} \times-i \mathbb{R}^{+} .
$$

Consequently,

$$
A \exp (i \Omega) \exp (i \Lambda) \cdot x_{0}=\{(z, w) \in \operatorname{Cr}(X) \mid \arg (w)=-\pi+\arg (z)\},
$$

and finally

as asserted.

$$
N A \exp (i \Omega) \exp (i \Lambda) \cdot x_{0}=\operatorname{Cr}(X)
$$

2.3. Mixed model for $\mathrm{SU}(2,1)$. Let $G=\mathrm{SU}(2,1)$ and $G_{\mathbb{C}}=\mathrm{Sl}(3, \mathbb{C})$. We let $G_{\mathbb{C}}$ act on $\mathbb{P}^{2}(\mathbb{C})=\left(\mathbb{C}^{3} \backslash\{0\} / \sim\right)$ by projectivized linear transformations. We embed $\mathbb{C}^{2}$ into $\mathbb{P}^{2}(\mathbb{C})$ via $z \mapsto[z, 1]$. Then $G$ preserves the ball

$$
X=\left\{z \in \mathbb{C}^{2} \mid\|z\|_{2}<1\right\} \simeq G / K,
$$

with the maximal compact subgroup $K=S(U(2) \times U(1))$ stabilizing the origin $x_{0}=0 \in X$. Note that an element

$$
g=\left(\begin{array}{cc}
A & u \\
v^{t} & \alpha
\end{array}\right) \in G
$$

with $u, v \in \mathbb{C}^{2}$, acts on $z \in X$ by

$$
g(z)=\frac{A z+u}{v^{t} \cdot z+\alpha}
$$

Let $\tau: G_{\mathbb{C}} \rightarrow G_{\mathbb{C}}$ be the holomorphic involution with fixed point set $\operatorname{SO}(2,1 ; \mathbb{C})$.

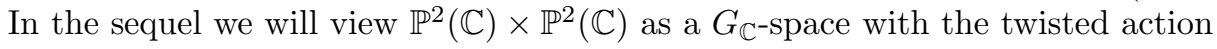

$$
g \cdot(z, w)=(g(z), \tau(g)(w)) \quad\left(z, w \in \mathbb{P}^{2}(\mathbb{C}) ; g \in G_{\mathbb{C}}\right) .
$$

Now, as $X$ is Hermitian, the crown is given by the double (see [13], Th. 7.7)

$$
\operatorname{Cr}(X)=X \times X
$$

but with $X$ embedded in $\operatorname{Cr}(X)$ as $z \mapsto(z, \bar{z})$. Note that this embedding is $G$ equivariant. Let

$$
A=\left\{a_{t}:=\left(\begin{array}{ccc}
\cosh t & 0 & \sinh t \\
0 & 1 & 0 \\
\sinh t & 0 & \cosh t
\end{array}\right) \mid t \in \mathbb{R}\right\},
$$

set $Y_{t}:=\log a_{t}$, and note that

$$
\Omega=\left\{Y_{t} \mid-\pi / 4<t<\pi / 4\right\}
$$


According to [10], Th. 8.11, we have

$$
\begin{gathered}
\Lambda=\left\{Z_{a, b}:=\left(\begin{array}{ccc}
i b & a & -i b \\
-\bar{a} & 0 & \bar{a} \\
i b & a & -i b
\end{array}\right) \mid a \in \mathbb{C}, b \in \mathbb{R} ;\right. \\
\left.|a|^{2}+|b|<1 / 2\right\} .
\end{gathered}
$$

We now define a subset $\mathcal{D} \subset \mathfrak{a} \times \mathfrak{n}$ by

$$
\mathcal{D}:=\left\{\left(Y_{t}, Z_{a, b}\right) \in \mathfrak{a} \times\left.\mathfrak{n}\left|\left(1-2|a|^{2}-2|b|\right) \cos (2 t)>(1-\cos (2 t))\right| a\right|^{2}\right\}_{0} .
$$

If $\pi_{\mathfrak{a}}: \mathfrak{a} \times \mathfrak{n} \rightarrow \mathfrak{a}$ denotes the first coordinate projection and $\pi_{\mathfrak{n}}$ resp. the second, we record:

$$
\begin{aligned}
& \text { - } \mathcal{D} \subset \Omega \times \Lambda \text {, } \\
& \text { - } \pi_{\mathfrak{a}}(\mathcal{D})=\Omega \text {, } \\
& \text { - } \pi_{\mathfrak{n}}(\mathcal{D})=\Lambda .
\end{aligned}
$$

In the coordinates $(2 a, 2 b, 2 t)$ (with $a$ real) we can visualize $\mathcal{D}$ as the interior of the following solid in $\mathbb{R}^{3}$ :

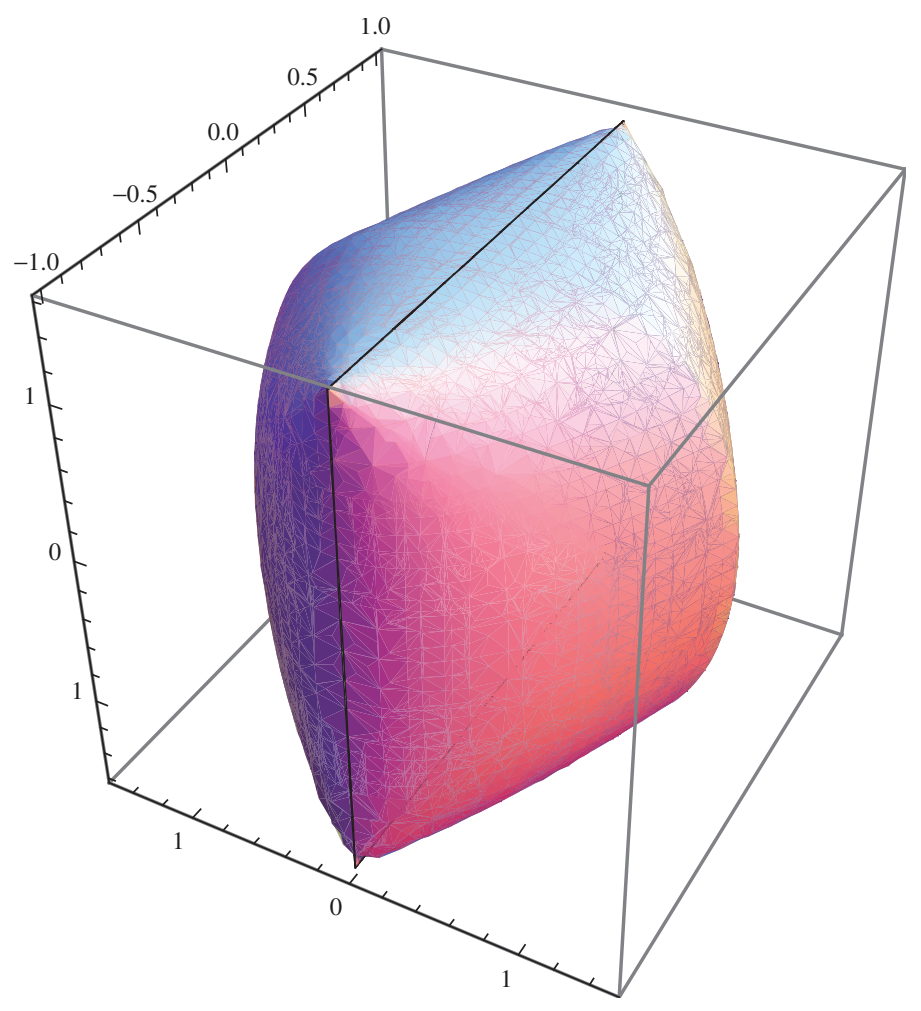

Proposition 2.2. Let $G=\mathrm{SU}(2,1)$. Then the map

$$
\Phi: N A \times \mathcal{D} \rightarrow \operatorname{Cr}(X), \quad(n a,(Y, Z)) \mapsto n a \exp (i Y) \exp (i Z) \cdot x_{0}
$$

is a diffeomorphism. 
Proof. All that we have to show is that the map is defined and onto.

To begin with we show that $\Phi$ is defined, i.e., $\operatorname{Im} \Phi \subset \operatorname{Cr}(X)$. Set $n_{a, b}:=$ $\exp \left(i Z_{a, b}\right)$. Let $M=Z_{K}(A)$. By $M$-invariance it is no loss of generality to assume that $a$ is real. Then

$$
n_{a, b}=\left(\begin{array}{ccc}
1-b+a^{2} / 2 & i a & b-a^{2} / 2 \\
-i a & 1 & i a \\
-b+a^{2} / 2 & i a & 1+b-a^{2} / 2
\end{array}\right) .
$$

We have to show that:

$$
a_{i \phi} n_{a, b}(0) \in X \quad \text { and } \quad \tau\left(a_{i \phi} n_{a, b}\right)(0) \in X
$$

for all parameters $(\phi, a, b) \in \mathcal{D}$. Note that $\tau\left(a_{i \phi}\right)=a_{i \phi}$ and $\tau\left(n_{a, b}\right)=n_{a,-b}$. Now

$$
n_{a, b}(0)=\frac{1}{1+b-a^{2} / 2}\left(b-a^{2} / 2, i a\right) .
$$

Note that $n_{a, b}(0) \in X$ if and only if

$$
\left(b-a^{2} / 2\right)^{2}+a^{2}<\left(1+b-a^{2} / 2\right)^{2}
$$

or

$$
-2 b+2 a^{2}<1 .
$$

Combined with $n_{a,-b}(0) \in X$ we arrive at $2|b|+2 a^{2}<1$, which is the defining condition of $\Lambda$.

We move on and apply $a_{i \phi}$ to $n_{a, \pm b}(0)$, and obtain that

$$
a_{i \phi} n_{a, \pm b}(0)=\frac{\left(\cos \phi \frac{ \pm b-a^{2} / 2}{1 \pm b-a^{2} / 2}+i \sin \phi, \frac{i a}{1 \pm b-a^{2} / 2}\right)}{\cos \phi+i \sin \phi \frac{ \pm b-a^{2} / 2}{1 \pm b-a^{2} / 2}} .
$$

Hence $a_{i \phi} n_{a, \pm b}(0) \in X$ if and only if

$$
\begin{aligned}
\cos ^{2} \phi+\sin ^{2} \phi \frac{\left( \pm b-a^{2} / 2\right)^{2}}{\left(1 \pm b-a^{2} / 2\right)^{2}}> & \cos ^{2} \phi \frac{\left( \pm b-a^{2} / 2\right)^{2}}{\left(1 \pm b-a^{2} / 2\right)^{2}}+ \\
& +\sin ^{2} \phi+\frac{a^{2}}{\left(1 \pm b-a^{2} / 2\right)^{2}}
\end{aligned}
$$

or, equivalently, after clearing denominators,

$$
\begin{array}{r}
\left(1 \pm b-a^{2} / 2\right)^{2} \cos ^{2} \phi+\left( \pm b-a^{2} / 2\right)^{2} \sin ^{2} \phi>\left( \pm b-a^{2} / 2\right)^{2} \cos ^{2} \phi \\
+\left(1 \pm b-a^{2} / 2\right)^{2} \sin ^{2} \phi+a^{2} .
\end{array}
$$

Simplifying further we arrive at

$$
\cos ^{2} \phi+2\left( \pm b-a^{2} / 2\right) \cos ^{2} \phi>\sin ^{2} \phi+2\left( \pm b-a^{2} / 2\right) \sin ^{2} \phi+a^{2}
$$

or, equivalently,

$$
\left(1 \pm 2 b-2 a^{2}\right) \cos (2 \phi)>(1-\cos 2 \phi) a^{2} .
$$

However, this is the defining condition for $\mathcal{D}$.

To see that the map is onto we observe that $\operatorname{Cr}(X) \subset N_{\mathbb{C}} A_{\mathbb{C}} \cdot x_{0}$. Hence there exists a domain $\mathcal{D}^{\prime} \subset \mathfrak{a}+\mathfrak{n}$ such that

$$
\operatorname{Cr}(X)=N A \cdot\left\{\exp (i Y) \exp (i Z) \mid(Y, Z) \in \mathcal{D}^{\prime}\right\} .
$$

If $\mathcal{D}^{\prime}$ is strictly larger than $\mathcal{D}$, then $\mathcal{D}^{\prime}$ would contain a boundary point of $\mathcal{D}$. Our computations show that this is not possible, and the proof of the proposition is concluded. 


\section{THE CROWN FOR HOMOGENEOUS HARMONIC SPACES}

Let $S$ be a simply connected noncompact homogeneous harmonic space. According to [7, Corollary 1.2, there are the following possibilities for $S$ :

(i) $S=\mathbb{R}^{n}$.

(ii) $S$ is the $A N$-part of a noncompact simple Lie group $G$ of real rank one.

(iii) $S=A \ltimes N$ with $N$ a nilpotent group of Heisenberg-type and $A \simeq \mathbb{R}$ acting on $N$ by graduation preserving scalings.

The case of $S=\mathbb{R}^{n}$ we will not consider; groups under (ii) are referred to as symmetric solvable harmonic groups. We mention that all spaces in (ii), except for those associated to $G=\mathrm{SO}_{o}(1, n)$, are of the type in (iii). Most issues of the Lorentz groups $G=\mathrm{SO}_{o}(1, n)$ readily reduce to $\mathrm{SO}_{o}(1,2) \simeq \operatorname{PSl}(2, \mathbb{R})$, where comprehensive treatments are available. In fact, for the real hyperbolic spaces $X=$ $H^{n}(\mathbb{R})=\mathrm{SO}_{o}(1, n) / \mathrm{SO}(n)$ we obtain the following result, analogous to Proposition 2.1 .

Proposition 3.1. Let $G=\mathrm{SO}_{o}(1, n)(n \geq 2)$. Then the map

$$
N A \times \Omega \times \Lambda \rightarrow \operatorname{Cr}(X), \quad(n a, H, Y) \mapsto n a \exp (i H) \exp (i Y) \cdot x_{0}
$$

is a diffeomorphism.

We will now focus on type (iii). In the sequel we recall some basic facts about $H$-type groups and their solvable harmonic extensions. We refer to 14 for a more comprehensive treatment and references. After that we introduce the crown domain for such harmonic extensions.

3.1. H-type Lie algebras and groups. Let $\mathfrak{n}$ be a real nilpotent Lie algebra of step two (that is, $[\mathfrak{n}, \mathfrak{n}] \neq\{0\}$ and $[\mathfrak{n},[\mathfrak{n}, \mathfrak{n}]]=\{0\}$ ), equipped with an inner product $\langle\cdot, \cdot\rangle$ and associated norm $|\cdot|$. Let $\mathfrak{z}$ be the center of $\mathfrak{n}$ and $\mathfrak{v}$ its orthogonal complement in $\mathfrak{n}$. Then

$$
\mathfrak{n}=\mathfrak{v} \oplus \mathfrak{z}, \quad[\mathfrak{v}, \mathfrak{z}]=0, \quad[\mathfrak{n}, \mathfrak{n}]=[\mathfrak{v}, \mathfrak{v}] \subset \mathfrak{z} .
$$

For $Z \in \mathfrak{z}$ let $J_{Z}: \mathfrak{v} \rightarrow \mathfrak{v}$ be the linear map defined by

$$
\left\langle J_{Z} V, V^{\prime}\right\rangle=\left\langle Z,\left[V, V^{\prime}\right]\right\rangle, \quad \forall V, V^{\prime} \in \mathfrak{v} .
$$

Then $\mathfrak{n}$ is called a Heisenberg-type algebra (or H-type algebra, for short) if

$$
J_{Z}^{2}=-|Z|^{2} \operatorname{id}_{\mathfrak{v}} \quad(Z \in \mathfrak{z})
$$

A connected and simply connected Lie group $N$ is called an H-type group if its Lie algebra $\mathfrak{n}=\operatorname{Lie}(N)$ is an H-type algebra; see 9 .

We let $p=\operatorname{dim} \mathfrak{v}, q=\operatorname{dim} \mathfrak{z}(\geq 1)$. Condition (3.1) implies that $p$ is even and $[\mathfrak{v}, \mathfrak{v}]=\mathfrak{z}$.

Moreover, (3.1) implies that the map $Z \rightarrow J_{Z}$ extends to a representation of the real Clifford algebra $\mathrm{Cl}(\mathfrak{z}) \cong \mathrm{Cl}_{q}$ on $\mathfrak{v}$. This procedure can be reversed and yields a general method for constructing $H$-type algebras.

Since $\mathfrak{n}$ is nilpotent, the exponential map exp : $\mathfrak{n} \mapsto N$ is a diffeomorphism. The Campbell-Hausdorff formula implies the following product law in $N$ :

$$
\exp X \cdot \exp X^{\prime}=\exp \left(X+X^{\prime}+\frac{1}{2}\left[X, X^{\prime}\right]\right), \quad \forall X, X^{\prime} \in \mathfrak{n} .
$$

This is sometimes written as

$$
(V, Z) \cdot\left(V^{\prime}, Z^{\prime}\right)=\left(V+V^{\prime}, Z+Z^{\prime}+\frac{1}{2}\left[V, V^{\prime}\right]\right)
$$


using the exponential chart to parametrize the elements $n=\exp (V+Z)$ by the couples $(V, Z) \in \mathfrak{v} \oplus \mathfrak{z}=\mathfrak{n}$.

3.1.1. Reduction theory. We conclude this section with reduction theory for $H$-type Lie algebras to Heisenberg algebras.

Let $\mathfrak{z}_{1}=\mathbb{R} Z_{1}$ be a one-dimensional subspace of $\mathfrak{z}$ and $\mathfrak{z}_{1}^{\perp}$ be its orthogonal complement in $\mathfrak{z}$. We assume that $\left|Z_{1}\right|=1$ and set $J_{1}:=J_{Z_{1}}$. We form the quotient algebra

$$
\mathfrak{n}_{1}:=\mathfrak{n} / \mathfrak{z}_{1}^{\perp}
$$

and record that $\mathfrak{n}_{1}$ is two-step nilpotent. Let $p_{1}: \mathfrak{z} \rightarrow \mathfrak{z}_{1}$ be the orthogonal projection. If we identify $\mathfrak{n}_{1}$ with the vector space $\mathfrak{v} \oplus \mathfrak{z}_{1}$ via the linear map

$$
\mathfrak{n}_{1} \rightarrow \mathfrak{v} \oplus \mathfrak{z}_{1}, \quad(V, Z)+\mathfrak{z}_{1}^{\perp} \mapsto\left(V, p_{1}(Z)\right),
$$

then the bracket in $\mathfrak{n}_{1}$ becomes in the new coordinates

$$
\left[\left(V, Z_{1}\right),\left(V^{\prime}, Z_{1}\right)\right]=\left(0,\left\langle Z_{1},\left[V, V^{\prime}\right]\right\rangle Z_{1}\right),
$$

where $V, V^{\prime} \in \mathfrak{v}$. Since $\left\langle Z_{1},\left[V, V^{\prime}\right]\right\rangle=\left\langle J_{1} V, V^{\prime}\right\rangle$, we see that $J_{1}$ determines a Lie algebra automorphism of $\mathfrak{n}_{1}$ and thus $\mathfrak{n}_{1}$ is isomorphic to the $p+1$-dimensional Heisenberg algebra.

3.2. Harmonic solvable extensions of $H$-type groups. Let $\mathfrak{n}$ be an H-type algebra with associated H-type group $N$. Let $\mathfrak{a}$ be a one-dimensional Lie algebra with an inner product. Write $\mathfrak{a}=\mathbb{R} H$, where $H$ is a unit vector in $\mathfrak{a}$. Let $A=\exp \mathfrak{a}$ be a one-dimensional Lie group with Lie algebra $\mathfrak{a}$ and isomorphic to $\mathbb{R}^{+}$(the multiplicative group of positive real numbers). Let the elements $a_{t}=\exp (t H) \in A$ act on $N$ by the dilations $(V, Z) \rightarrow\left(e^{t / 2} V, e^{t} Z\right)$ for $t \in \mathbb{R}$, and let $S$ be the associated semi-direct product of $N$ and $A$ :

$$
S=N A=N \rtimes A .
$$

The action of $A$ on $N$ becomes the inner automorphism

$$
a_{t} \exp (V+Z) a_{t}^{-1}=\exp \left(e^{t / 2} V+e^{t} Z\right),
$$

and the product in $S$ is given by

$$
\exp (V+Z) a_{t} \exp \left(V^{\prime}+Z^{\prime}\right) a_{t^{\prime}}=\exp (V+Z) \exp \left(e^{t / 2} V^{\prime}+e^{t} Z^{\prime}\right) a_{t+t^{\prime}} .
$$

$S$ is a connected and simply connected Lie group with Lie algebra

$$
\mathfrak{s}=\mathfrak{n} \oplus \mathfrak{a}=\mathfrak{v} \oplus \mathfrak{z} \oplus \mathfrak{a}
$$

and Lie bracket defined by linearity and the requirement that

$$
[H, V]=\frac{1}{2} V, \quad[H, Z]=Z, \quad \forall V \in \mathfrak{v}, \forall Z \in \mathfrak{z} .
$$

The map $(V, Z, t H) \rightarrow \exp (V+Z) \exp (t H)$ is a diffeomorphism of $\mathfrak{s}$ onto $S$. If we parametrize the elements $n a=\exp (V+Z) \exp (t H) \in N A$ by the triples $(V, Z, t) \in \mathfrak{v} \times \mathfrak{z} \times \mathbb{R}$, then the product law reads

$$
(V, Z, t) \cdot\left(V^{\prime}, Z^{\prime}, t^{\prime}\right)=\left(V+e^{t / 2} V^{\prime}, Z+e^{t} Z^{\prime}+\frac{1}{2} e^{t / 2}\left[V, V^{\prime}\right], t+t^{\prime}\right),
$$

for all $V, V^{\prime} \in \mathfrak{v}, Z, Z^{\prime} \in \mathfrak{z}, t, t^{\prime} \in \mathbb{R}$. For $n=(V, Z, 0) \in N$ and $a_{t}=(0,0, t) \in A$ we consistently get $n a_{t}=(V, Z, t)$. 
We extend the inner products on $\mathfrak{n}$ and $\mathfrak{a}$ to an inner product $\langle\cdot, \cdot\rangle$ on $\mathfrak{s}$ by linearity and the requirement that $\mathfrak{n}$ be orthogonal to $\mathfrak{a}$. The left-invariant Riemannian metric on $S$ defined by this inner product turns $S$ into a harmonic solvable group [5, 6].

3.3. The complexification and the crown. Let $N_{\mathbb{C}}$ be the simply connected Lie group with Lie algebra $\mathfrak{n}_{\mathbb{C}}$ and set $A_{\mathbb{C}}=\mathbb{C}^{*}$. Then $A_{\mathbb{C}}$ acts on $N_{\mathbb{C}}$ by $t \cdot(V, Z):=$ $\left(t V, t^{2} Z\right)$ for $t \in \mathbb{C}^{*}$ and $(V, Z) \in N_{\mathbb{C}}$.

We define a complexification $S_{\mathbb{C}}$ of $S$ by

$$
S_{\mathbb{C}}=N_{\mathbb{C}} \rtimes A_{\mathbb{C}} .
$$

We identify the Lie algebra $\mathfrak{a}$ with $\mathbb{R}$ by sending $H \mapsto 1 / 2$. For $z \in \mathbb{C}$ we often set $a_{z}:=\exp (z H)$ and note that $a_{z}$ corresponds to $e^{z / 2} \in \mathbb{C}^{*}$. In particular,

$$
\{z \in \mathbb{C}: \exp (z H)=e\}=4 \pi i \mathbb{Z} .
$$

It follows that the exponential map exp $: \mathfrak{a}_{\mathbb{C}} \rightarrow A_{\mathbb{C}}$ is certainly injective if restricted to $\mathfrak{a} \oplus i H(-2 \pi, 2 \pi]$.

Motivated by our discussion of $\mathrm{SU}(2,1)$ and the discussed $\mathrm{SU}(n, 1)$-reduction, we define the following sets for a more general harmonic $A N$-group:

$$
\begin{gathered}
\Omega=\left\{t H \in \mathfrak{a}:|t|<\frac{\pi}{2}\right\}, \\
\Lambda=\left\{(V, Z) \in \mathfrak{n}: \quad \frac{1}{2}|V|^{2}+|Z|<1\right\}, \\
\mathcal{D}=\left\{(V, Z, t) \in \mathfrak{s}: \cos t\left(1-\frac{1}{2}|V|^{2}-|Z|\right)>\frac{1}{4}(1-\cos t)|V|^{2}\right\}_{0}, \\
D=\{\exp (i t H) \exp (i V+i Z):(V, Z, t) \in \mathcal{D}\} \subset S_{\mathbb{C}}
\end{gathered}
$$

Here as usual $\{\cdot\}_{0}$ denotes the connected component of $\{\cdot\}$ containing 0 , and we write $(V, Z, t)$ for the element $V+Z+t H$ of $\mathfrak{s}$.

Definition 3.2. The complex crown of $S$ will be defined as the following subset of $S_{\mathbb{C}}:$

$$
\operatorname{Cr}(S)=N A D \subset N A \exp (i \Omega) \exp (i \Lambda) \subset S_{\mathbb{C}} .
$$

It is easy to see that $\operatorname{Cr}(S)$ is open and simply connected in $S_{\mathbb{C}}$.

We conclude with the proof of the mixed model of the crown for rank one symmetric spaces. In this case we let $S=A N$ be the solvable part of the rank one group $G=N A K$. Note that $F:=A_{\mathbb{C}} \cap K_{\mathbb{C}}$ is isomorphic to $\mathbb{Z}_{2}$ and that there is

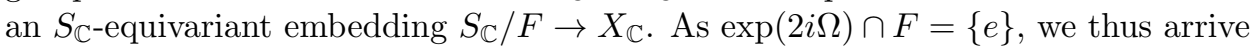
at an $S$-equivariant embedding $\operatorname{Cr}(S) \hookrightarrow X_{\mathbb{C}}$.

Theorem 3.3. Let $X=G / K$ be a noncompact Riemannian symmetric space of rank one, $X \neq H^{n}(\mathbb{R})$. Let $S=N A$, and let $\operatorname{Cr}(S)$ be embedded in $X_{\mathbb{C}}$ by $z \mapsto z \cdot x_{0}$. Then

$$
\operatorname{Cr}(X)=\operatorname{Cr}(S) .
$$

Proof. We first show that $\operatorname{Cr}(S) \subset \operatorname{Cr}(X)$. We have to show that $\exp (i t H) \exp (i Y)$. $x_{0} \in \operatorname{Cr}(X)$ for all $(Y, t) \in \mathcal{D}$. Let $M=Z_{K}(A)$ and let $S_{\mathfrak{v}}, S_{\mathfrak{z}}$ denote the unit spheres in $\mathfrak{v}$ and $\mathfrak{z}$. Since the group $\operatorname{Ad}(M)$ acts transitively on $S_{\mathfrak{v}} \times S_{\mathfrak{z}}$ (see, e.g., [3], Theorem 6.2, for a simple proof within the framework of $H$-type groups), the assertion is reduced to $G=\mathrm{SU}(2,1)$, where it was shown in Proposition 2.2 above.

For the converse inclusion let $X_{1}=G_{1} / K_{1} \subset X=G / K$ be a subdomain with $K_{1} \subset K$ and $\Omega_{1}=\Omega$. We claim that $\partial \operatorname{Cr}\left(X_{1}\right) \subset \partial \operatorname{Cr}(X)$. In fact, if $z \in \partial \operatorname{Cr}\left(X_{1}\right)$, then Lemma 2.3 (ii) in [13] shows that $z$ is the limit of a sequence 
$x_{n}:=g_{n} \exp \left(i Y_{n}\right) \cdot x_{0}$ with $g_{n} \in G_{1}$ and $\Omega_{1} \ni Y_{n} \rightarrow Y \in \partial \Omega_{1}$. On the other hand, assuming $z \in \operatorname{Cr}(X)$ we would also have $z=g \exp (i Z) \cdot x_{0}$ with $g \in G$ and $Z \in \Omega$. By following the proof of the cited lemma one sees that $g^{-1} g_{n}$ must be bounded, from which a contradiction is easily reached. Hence $z \in \partial \operatorname{Cr}(X)$.

In order to conclude the proof of the theorem, it is enough to show that the elements which are in the boundary of $D$ do not lie in $\operatorname{Cr}(X)$, i.e.,

$$
\{\exp (i t H) \exp (i V+i Z):(V, Z, t) \in \partial \mathcal{D}\} \cap \operatorname{Cr}(X)=\emptyset .
$$

This again reduces to $G=\mathrm{SU}(2,1)$ and thus follows from $\partial \operatorname{Cr}\left(X_{1}\right) \subset \partial \operatorname{Cr}(X)$.

\section{Geometric analysis}

Let $S$ be a simply connected noncompact homogeneous harmonic space, $S \neq \mathbb{R}^{n}$. In this section we will show that the eigenfunctions of the Laplace-Beltrami operator on $S$ extend holomorphically to $\operatorname{Cr}(S)$ and that $\operatorname{Cr}(S)$ is maximal with respect to this property.

4.1. Holomorphic extension of eigenfunctions. For $z \in D$ we consider the following totally real embedding of $S$ into $\operatorname{Cr}(S)$ :

$$
S \hookrightarrow \operatorname{Cr}(S), \quad s \mapsto s z .
$$

Now let $\mathcal{L}$ be the Laplace-Beltrami operator on $S$, explicitly given by (see [14])

$$
\mathcal{L}:=\sum_{j=1}^{p} V_{j}^{2}+\sum_{i=1}^{q} Z_{i}^{2}+H^{2}-2 \rho H,
$$

where $2 \rho=(p / 2)+q$ and the $\left(V_{j}\right)_{j}$ and $\left(Z_{i}\right)_{i}$ form an orthonormal basis of $\mathfrak{v}$ and $\mathfrak{z}$, respectively. Here we consider the elements $X \in \mathfrak{s}$ as left-invariant vector fields on $S$. Hence it is clear that $\mathcal{L}$ extends to a left $S_{\mathbb{C} \text {-invariant holomorphic differential }}$ operator on $S_{\mathbb{C}}$, which we denote by $\mathcal{L}_{\mathbb{C}}$. Now if $M \subset S_{\mathbb{C}}$ is a totally real analytic submanifold, then we can restrict $\mathcal{L}_{\mathbb{C}}$ to $M$, in symbols $\mathcal{L}_{M}$, in the following way: if $f$ is a real analytic function near $m \in M$ and $f_{\mathbb{C}}$ is a holomorphic extension of $f$ in a complex neighborhood of $m$ in $S_{\mathbb{C}}$, then set

$$
\left(\mathcal{L}_{M} f\right)(m):=\left(\mathcal{L}_{\mathbb{C}} f_{\mathbb{C}}\right)(m) .
$$

Then:

Proposition 4.1. For all $z \in D$ the restriction $\mathcal{L}_{S z}$ is elliptic.

Proof. To illustrate what is going on we first give a proof for those $S$ related to $G=\operatorname{Sl}(2, \mathbb{R})$. We consider this as the degenerate case where $\mathfrak{v}=\{0\}$ so that $\operatorname{ad}(H)$ acts on $\mathfrak{n}=\mathfrak{z}=\mathbb{R} Z$ as the identity map (see [3], section 3(d)). Here

$$
H=\left(\begin{array}{cc}
1 / 2 & 0 \\
0 & -1 / 2
\end{array}\right), \quad Z=\left(\begin{array}{ll}
0 & 1 \\
0 & 0
\end{array}\right) .
$$

The set $\mathcal{D}$ degenerates to the product

$$
\mathcal{D}=\Omega \times \Lambda=\left\{t H:|t|<\frac{\pi}{2}\right\} \times\{x Z:|x|<1\},
$$

and $D=\exp (i \Omega) \exp (i \Lambda)$, in agreement with Proposition 2.1. The Laplace-Beltrami operator on $S$ becomes $\mathcal{L}=Z^{2}+H^{2}-H$. Let $z=\exp (i t H) \exp (i x Z) \in D$. Using 
$[H, Z]=Z$ we get

$$
\begin{aligned}
\operatorname{Ad}(z) H & =H-i e^{i t} x Z, \\
\operatorname{Ad}(z) Z & =e^{i t} Z .
\end{aligned}
$$

It follows that the leading symbol, or principal part, of $\mathcal{L}_{S z}$ is given by

$$
\left[\mathcal{L}_{S z}\right]_{\text {prin }}=\left(H-i e^{i t} x Z\right)^{2}+e^{2 i t} Z^{2} .
$$

Let us verify that $\mathcal{L}_{S z}$ is elliptic. The associated quadratic form is given by the matrix

$$
L(z):=\left(\begin{array}{cc}
1 & -i x e^{i t} \\
-i x e^{i t} & e^{2 i t}\left(1-x^{2}\right)
\end{array}\right) .
$$

We have to show that $\langle L(z) \xi, \xi\rangle=0$ has no solution for $\xi=\left(\xi_{1}, \xi_{2}\right) \in \mathbb{R}^{2} \backslash\{0\}$. We look at

$$
\xi_{1}^{2}-2 i x e^{i t} \xi_{1} \xi_{2}+e^{2 i t}\left(1-x^{2}\right) \xi_{2}^{2}=0 .
$$

Now $\xi_{2}=0$ is readily excluded, and we remain with the quadric

$$
\xi^{2}-2 i x e^{i t} \xi+e^{2 i t}\left(1-x^{2}\right)=0,
$$

whose solutions are

$$
\begin{aligned}
\lambda_{1,2} & =i x e^{i t} \pm \sqrt{-x^{2} e^{2 i t}-\left(1-x^{2}\right) e^{2 i t}} \\
& =i e^{i t}(x \pm 1) .
\end{aligned}
$$

These are never real in the domain $-\frac{\pi}{2}<t<\frac{\pi}{2}$ and $-1<x<1$. The same proof works for those $S$ related to $\mathrm{SO}_{o}(1, n), n \geq 2$.

To put the computation above in a more abstract framework: it is to show here that for $z \in D$ the operator $L(z): \mathfrak{s}_{\mathbb{C}} \rightarrow \mathfrak{s}_{\mathbb{C}}$ defined by

$$
L(z):=\operatorname{Ad}(z)^{t} \operatorname{Ad}(z)
$$

is elliptic in the sense that $\langle L(z) \xi, \xi\rangle=0$ for $\xi \in \mathfrak{s}$ implies $\xi=0$.

Thus, for the sequel we assume that $\mathfrak{n}$ is $H$-type, i.e., $\mathfrak{v}$ and $\mathfrak{z}$ are $\neq\{0\}$. We will reduce to the case where $N$ is a Heisenberg group, i.e., $S$ is related to $\mathrm{SU}(1, n)$. We use the already introduced technique of reduction to Heisenberg groups. Therefore, let $Z_{1} \in \mathfrak{z}$ be a normalized element and $\mathfrak{n}_{1}=\mathfrak{n} / \mathfrak{z}_{1}^{\perp}$ be as before. We let $S_{1}$ be the harmonic group associated to $N_{1}$ and note that there is a natural group homomorphism $S \rightarrow S_{1}$ which extends to a holomorphic map $S_{\mathbb{C}} \rightarrow\left(S_{1}\right)_{\mathbb{C}}$ which maps $\operatorname{Cr}(S)$ onto $\operatorname{Cr}\left(S_{1}\right)$. Now the assertion is true for $\operatorname{Cr}\left(S_{1}\right)$ in view of [11] (proof of Th. 3.2) and Theorem 3.3. Let $M$ be the group of automorphisms of $S$ preserving the inner product on $\mathfrak{s}$. By [3], Proposition 6.1 and Remark 6.3, the group $\operatorname{Ad}(M)$ acts transitively on the unit sphere in $\mathfrak{z}$. Thus the ellipticity of (4.1) is true for $S$ if it is true for all choices of $S_{1}$, and the assertion follows.

As a consequence of this fact, we obtain as in [1] that:

Theorem 4.2. Every $\mathcal{L}$-eigenfunction on $S$ extends to a holomorphic function on $\operatorname{Cr}(S)$.

Proof (analogous to the proof of Th. 3.2 of [11]). As $\mathcal{L}$ is elliptic, the regularity theorem for elliptic differential operators implies that all eigenfunctions $f$ are analytic and extend holomorphically on a neighborhood of $\mathbf{1}$ in $S_{\mathbb{C}}$. This neighborhood can be chosen independently of $f$. 
Let $0 \leq t \leq 1$ and define $\mathcal{D}_{t}:=t \mathcal{D}$ and correspondingly $D_{t}$. It follows that all eigenfunctions $f$ extend holomorphically to a common domain of the form $U D_{t} \subset$ $S_{\mathbb{C}}$ for some $0<t \leq 1$ and some open neighborhood $U$ of $\mathbf{1}$ in $S$. As $\mathcal{L}$ is $S$-invariant we can replace $f$ by any left $S$-translate and conclude that $f$ extends to $S D_{t} \subset S_{\mathbb{C}}$.

If $t=1$, we are finished. Otherwise, let $t$ be such that all eigenfunctions $f$ extend to $S D_{t}$, but for each $t<t^{\prime} \leq 1$ some eigenfunction does not extend to $S D_{t^{\prime}}$. Let $(Y, r) \in \partial \mathcal{D}_{t}$ and let $z=\exp (i r H) \exp (i Y)$. By our previous proposition, $\mathcal{L}_{S z}$ is elliptic. Now it comes down to choosing appropriate local coordinates to see that $f$ extends holomorphically on a complex cone based at $z$. The condition (9.4.16) in $[8$, Cor. 9.4.9, is verified so that this corollary applies. We conclude that there exists an open neighborhood of $z$ on which all eigenfunctions $f$ are holomorphic. By compactness of $\partial D_{t}$ we then find $t^{\prime}>t$ and an open neighborhood $U$ of $\mathbf{1}$ in $S$, both independent of $f$, such that $f$ extends holomorphically to $U D_{t^{\prime}}$. As before we obtain that $f$ extends to $S D_{t^{\prime}}$. As this is valid for all $f$ a contradiction is reached and the theorem follows.

4.2. Maximality of $\operatorname{Cr}(S)$. We begin with a collection of some facts about Poisson kernels on $S$. Let us denote by $\theta: S \rightarrow S$ the geodesic symmetry, centered at the identity. Every $z \in S_{\mathbb{C}}$ can be uniquely written as $z=n(z) a(z)$ with $n \in N_{\mathbb{C}}$ and $a \in A_{\mathbb{C}}$. As $\operatorname{Cr}(S)$ is simply connected, we obtain for every $\lambda \in \mathfrak{a}_{\mathbb{C}}^{*}$ a holomorphic map

$$
a^{\lambda}: \operatorname{Cr}(S) \rightarrow \mathbb{C}, z \mapsto e^{\lambda \log a(z)} .
$$

The function $P_{\lambda}:=a^{\rho-i \lambda} \circ \theta$ on $S$ is referred to as Poisson kernel on $S$ with parameter $\lambda$. We note that both $a^{\lambda}$ and $P_{\lambda}$ are $\mathcal{L}$-eigenfunctions [4, 1].

For $\lambda \in \mathfrak{a}_{\mathbb{C}}^{*}$, we define the spherical function with parameter $\lambda$ by

$$
\phi_{\lambda}(s):=b \int_{N} P_{\lambda}(n s) P_{-\lambda}(n) d n \quad(s \in S),
$$

where $b=2^{q-1} \Gamma\left(\frac{p+q+1}{2}\right) / \pi^{\frac{p+q+1}{2}}$ is a constant such that $\phi_{\lambda}(e)=1$.

Let us denote by ${ }^{2} \subset \mathfrak{a}_{\mathbb{C}}^{*}$ the parameter set for nontrivial positive definite spherical functions on $S$ and note that $\mathfrak{a}^{*} \subset \mathcal{X}$.

Theorem 4.3. The crown domain $\operatorname{Cr}(S)$ is the unique largest $S$-invariant domain in $S_{\mathbb{C}}$ containing $S$ with the property that all $\mathcal{L}$-eigenfunctions extend holomorphically to $\operatorname{Cr}(S)$.

Proof. Let us first discuss the case of symmetric $S$, i.e. $S \simeq X=G / K$ and $\operatorname{Cr}(S)=\operatorname{Cr}(X)$. Fix $\lambda \in \mathcal{X}$. In this case it was shown in Section 5 of [12] (with corrigendum in [10, Remark 4.8) that $\operatorname{Cr}(X)$ is the unique largest $G$-invariant domain in $X_{\mathbb{C}}$ containing $X$ to which $\phi_{\lambda}$ extends holomorphically. We will now adapt this method to the $S$-invariant situation.

Let $z \in \partial \operatorname{Cr}(S)$. Recall the elliptic boundary of $\operatorname{Cr}(X)$ in $X_{\mathbb{C}}, \partial_{\text {ell }} \operatorname{Cr}(X)=$ $G \exp (i \partial \Omega) \cdot x_{0} \subset \partial \operatorname{Cr}(X)$. Let us first assume that $z \in \partial \operatorname{Cr}(S) \cap \partial_{\text {ell }} \operatorname{Cr}(X)$. Assume that $\phi_{\lambda}$ extends holomorphically to an open neighborhood $U \subset S_{\mathbb{C}}$ of $z$. We find $r>1$ and $z_{0} \in \exp (i r \partial \Omega)$ and $g \in G$ such that $g z_{0} \in U$.

Set $\psi_{\lambda}:=\phi_{\lambda} \circ g^{-1}$. Then $\psi_{\lambda}$ is an $\mathcal{L}$-eigenfunction. Now it was shown in [12] (Lemma 5.3 and Th. 5.4 with proof) that there exists an $s \in S$ and a sequence $\left(s_{n}\right)_{n \in \mathbb{N}} \subset S$ with $\lim _{n \rightarrow \infty} s_{n}=s \in S$ such that

$$
0 \leq \phi_{\lambda}\left(s_{n} z_{0}\right) \nearrow \infty \text {. }
$$


Now Lemma 5.3 in 12 readily modifies and allows us to replace the sequence $\left(s_{n}\right)_{n \in N}$ by a sequence $\left(g^{-1} u_{n} g\right)_{n \in \mathbb{N}}$ with $u_{n} \in S$ converging to $u \in S$. Hence we get that

$$
0 \leq \psi_{\lambda}\left(u_{n} z\right) \nearrow \infty
$$

a contradiction. If $z$ is not in the elliptic boundary, then we argue as in [10, Rem. 4.8 , and reach the same conclusion.

For nonsymmetric $S$ we apply $\mathrm{SU}(2,1)$-reduction (see [2]). If $z \in \operatorname{Cr}(S)$, then we can put $z \in \operatorname{Cr}\left(S_{1}\right)$ with $\operatorname{Cr}\left(S_{1}\right)$ an $\mathrm{SU}(2,1)$-crown contained in $\operatorname{Cr}(S)$. Let $\mathcal{X}_{1}$ be the set of equivalence classes of nontrivial positive definite spherical functions on $S_{1}$ and note that $\mathcal{X}_{1}=\mathfrak{a}^{*} \amalg i\left(-c \rho_{1}, c \rho_{1}\right)$ for some $c>0$. Disintegration theory of unitary representations yields a positive Radon measure $\mu$ on $\mathcal{X}_{1}$ such that

$$
\left.\phi_{\lambda}\right|_{S_{1}}=\int_{\mathcal{X}_{1} \cup\{\mathbf{1}\}} \phi_{\sigma}^{1} d \mu(\sigma) .
$$

Here $\phi_{\sigma}^{1}$ stands for the spherical function on $S_{1}$ with parameter $\sigma$. If we can exclude the case that $\operatorname{supp} \mu=\{\mathbf{1}\}$, then the assertion follows with (4.2) as before.

Finally $\operatorname{supp} \mu=\{\mathbf{1}\}$ cannot happen, as $\left.\phi_{\lambda}\right|_{A}$ is not constant and $A \subset S_{1}$.

Corollary 4.4. The geodesic symmetry extends to a holomorphic involutive map $\theta: \operatorname{Cr}(S) \rightarrow \operatorname{Cr}(S)$.

Proof. For all $\lambda \in \mathfrak{a}_{\mathbb{C}}^{*}$ the Poisson kernel $P_{\lambda}=a^{\rho-i \lambda} \circ \theta$ extends to a holomorphic function on $\operatorname{Cr}(S)$. Thus the map $\theta$ must extend to a holomorphic involutive map

$$
\theta: \operatorname{Cr}(S) \rightarrow S_{\mathbb{C}}
$$

To see that $\theta(\operatorname{Cr}(S))=\operatorname{Cr}(S)$, we observe that $\theta(\operatorname{Cr}(S))$ is an open connected, $S$ invariant neighborhood of $S$ in $S_{\mathbb{C}}$ ( $\theta$ being holomorphic and involutive). Suppose this neighborhood is not contained in $\operatorname{Cr}(S)$. Then there exists a boundary point $z \in \partial \operatorname{Cr}(S)$ and a $q \in \operatorname{Cr}(S)$ such that $z=\theta(q)$. However, then

$$
P_{\lambda}(z)=a^{\rho-i \lambda}(q) \text {. }
$$

Now the right-hand side in this formula is well defined $\forall \lambda \in \mathfrak{a}_{\mathbb{C}}^{*}$, whereas the lefthand side blows up for some $\lambda \in \mathfrak{a}_{\mathbb{C}}^{*}$ (if it did not, then $\phi_{\lambda}$ for some $\lambda \in \mathcal{X}$ would extend holomorphically to an $S$-invariant domain strictly containing $\operatorname{Cr}(S)$ ). It follows that $\theta(\operatorname{Cr}(S)) \subseteq \operatorname{Cr}(S)$. The opposite inclusion is clear from the involutivity of $\theta$.

\section{ACKNOWLEDGEMENT}

The authors would like to thank the anonymous referee for the careful screening of the manuscript and for many useful suggestions.

\section{REFERENCES}

[1] J.-P. Anker, E. Damek, and C. Yacoub, Spherical analysis on harmonic AN groups, Ann. Scuola Norm. Sup. Pisa 23 (1996) 643-679. MR1469569 (99a:22014)

[2] M.G. Cowling, A.H. Dooley, A. Koranyi, and F. Ricci, H-type groups and Iwasawa decomposition, Adv. Math. 87 (1991) 1-41. MR.1102963 (92e:22017)

[3] M.G. Cowling, A.H. Dooley, A. Koranyi, and F. Ricci, An approach to symmetric spaces of rank one via groups of Heisenberg type, J. Geom. Anal. 8 (1998) 199-237. MR.1705176 (2000m:53071)

[4] E. Damek, A Poisson kernel on Heisenberg type nilpotent groups, Colloq. Math. 53 (1987) 239-247. MR924068 (89d:22006) 
[5] E. Damek and F. Ricci, A class of nonsymmetric harmonic Riemannian spaces, Bull. Amer. Math. Soc. 27 (1992) 139-142. MR1142682 (93b:53043)

[6] E. Damek and F. Ricci, Harmonic analysis on solvable extensions of $H$-type groups, J. Geom. Anal. 2 (1992) 213-248. MR.1164603 (93d:43006)

[7] J. Heber, On harmonic and asymptotically harmonic spaces, Geom. Funct. Anal. 16 (2006) 869-890. MR2255384(2007e:53060)

[8] L. Hörmander, The Analysis of Linear Partial Differential Operators I, Springer Grundlehren 256, 1983. MR717035 (85g:35002a)

[9] A. Kaplan, Fundamental solutions for a class of hypoelliptic PDE generated by composition of quadratic forms, Trans. Amer. Math. Soc. 258 (1980) 147-153. MR.554324 (81c:58059)

[10] B. Krötz and E. M. Opdam, Analysis on the crown domain, Geom. Funct. Anal. 18 (2008) 1326-1421. MR 2465692(2010a:22011)

[11] B. Krötz and H. Schlichtkrull, Holomorphic extension of eigenfunctions, Math. Ann. 345 (2009) 835-841. MR2545868 (2010k:22016)

[12] B. Krötz, G. Ólafsson and R.J. Stanton, The Image of the Heat Kernel Transform on Riemannian Symmetric Spaces of the Noncompact Type, Int. Math. Res. Not. 2005, no. 22, 1307-1329. MR 2152539 (2006e:58036)

[13] B. Krötz and R.J. Stanton, Holomorphic extensions of representations. II. Geometry and Harmonic Analysis, Geom. Funct. Anal. 15 (2005) 190-245. MR2140631 (2006d:43010)

[14] F. Rouviere, Espaces de Damek-Ricci, geometrie et analyse, Semin. Congr. 7, Soc. Math. France (2003) 45-100. MR2038648 (2004m:22015)

Dipartimento di Matematica, Politecnico di Torino, Corso Duca degli Abruzzi 24, 10129 TORINO, ITALY

E-mail address: camporesi@polito.it

Institut für Analysis, Leibniz Universität Hannover, Welfengarten 1, D-30167 HanNOVER, GERMANY

E-mail address: kroetz@math.uni-hannover.de 